TRANSACTIONS OF THE

AMERICAN MATHEMATICAL SOCIETY

Volume 349, Number 6, June 1997, Pages 2525-2536

S $0002-9947(97) 01733-9$

\title{
HERZ-SCHUR MULTIPLIERS AND WEAKLY ALMOST PERIODIC FUNCTIONS ON LOCALLY COMPACT GROUPS
}

\author{
GUANGWU XU
}

\begin{abstract}
For a locally compact group $G$ and $1<p<\infty$, let $A_{p}(G)$ be the Herz-Figà-Talamanca algebra and $B_{p}(G)$ the Herz-Schur multipliers of $G$, and $M A_{p}(G)$ the multipliers of $A_{p}(G)$. Let $W(G)$ be the algebra of continuous weakly almost periodic functions on $G$. In this paper, we show that (1), if $G$ is a noncompact nilpotent group or a noncompact [IN]-group, then $W(G) / B_{p}(G)^{-}$ contains a linear isometric copy of $l^{\infty}(\mathbb{N}) ;(2)$, for a noncommutative free group $F, B_{p}(F)$ is a proper subset of $M A_{p}(F) \cap W(F)$.
\end{abstract}

\section{INTRODUCTION}

Let $G$ be a locally compact group, $C(G)$ the space of bounded continuous functions on $G$ with the sup norm. For a subset $S$ of $C(G), S^{-}$denotes the uniform closure of $S$ in $C(G)$. Let $A_{p}(G)$ be the Herz-Figà-Talamanca algebra of $G$ and $B_{p}(G)$ the algebra of Herz-Schur multipliers, with $1<p<\infty$. Note that $A_{2}(G)=A(G)$ is the Fourier algebra of $G$, introduced by Eymard [12], and $B_{2}(G)$ is the completely bounded multipliers $M_{0} A(G)$ of $A(G)$, as was shown by Bożejko and Fendler [5]. The Fourier-Stieltjes algebra $B(G)$ of $G$ is the space of coefficients of strongly continuous unitary representations of $G$. It is known that $B(G) \subseteq M_{0} A(G)$, and they are equal if $G$ is amenable. Also, $M_{0} A(G) \subseteq B_{p}(G)$ for every $1<p<\infty$ (see [1], $[15])$. Let $W(G)$ be the algebra of continuous weakly almost periodic functions on $G$. Then it can be shown that $B_{p}(G) \subseteq W(G)$ for every $1<p<\infty$. In answering a question raised by Eberlein, i.e., whether for an abelian group $G, B(G)^{-}=W(G)$, Rudin [32] showed that $B(G)^{-} \subsetneq W(G)$ if $G$ is abelian and contains a discrete subgroup which is not of bounded order, and Ramirez [31] later showed that Rudin's conclusion holds for all noncompact abelian groups. More general results on this topic were obtained by Chou [7]. He extended the Rudin-Ramirez result to include many nonabelian groups: if $G$ is either a noncompact nilpotent group or a noncompact [IN]-group, then $W(G) / B(G)^{-}$contains a linear isometric copy of $l^{\infty}(\mathbb{N})$, in particular $B(G)^{-} \subsetneq W(G)$. In the first part of this paper, we are able to replace $B(G)$ by some larger spaces. More precisely, we have the following result: for every $1<p<\infty, W(G) / B_{p}(G)^{-}$contains a linear isometric copy of $l^{\infty}(\mathbb{N})$, if $G$ is a noncompact nilpotent group or a noncompact [IN]-group. This generalizes Chou's result mentioned above. This will be the contents of sections 3 and 4 .

Received by the editors November 29, 1994 and, in revised form, January 29, 1996.

1991 Mathematics Subject Classification. Primary 43A30, 43A60, 43A46; Secondary 22D05, $22 \mathrm{D} 25$. 
If $G$ is a locally compact group, we denote by $M A_{p}(G)$ the space of multipliers of $A_{p}(G), 1<p<\infty$. When $G$ is amenable, we have the equality $B_{p}(G)=M A_{p}(G)$. It was shown by Bo $\dot{z}$ ejko [2], [4] that for a noncommutative free group $F, B_{p}(F)$ is a proper subset of $M A_{p}(F)$. In fact he constructed a function $\phi$ in [4] such that $\phi \in M A_{p}(F)$ but $\phi \notin W(F)$, hence $\phi \notin B_{p}(F)$. It is therefore interesting to decide whether $B_{p}(F)=M A_{p}(F) \cap W(F)$. In section 5, by constructing a Leinert set and using the discussions in section 3 , we are able to show that $B_{p}(F)$ is a proper subset of $M A_{p}(F) \cap W(F)$ for a free group $F$ on at least two generators.

\section{Preliminaries}

Let $G$ be a locally compact group with a fixed left Haar measure and $L^{p}(G), 1 \leq$ $p \leq \infty$, the usual Lebesgue spaces on $G$ with the norm $\|\cdot\|_{p}$.

Suppose that $1<p<\infty$ and $\frac{1}{p}+\frac{1}{q}=1$. The Herz-Figà-Talamanca algebra $A_{p}(G)$ is the space of continuous functions $u$ which can be represented as

$$
u=\sum_{i=1}^{\infty} f_{i} * \check{g}_{i},
$$

where $f_{i} \in L^{q}(G), g_{i} \in L^{p}(G)\left(\check{g}_{i}(x)=g_{i}\left(x^{-1}\right)\right)$ and $\sum_{i=1}^{\infty}\left\|f_{i}\right\|_{p}\left\|g_{i}\right\|_{q}<\infty$, with norm the infimum of the last expression over all such representations of $u$. $A_{p}(G)$ is a Banach algebra with pointwise multiplication. Note that $A_{p}(G)$ is contained in $C_{0}(G)$, the subspace of $C(G)$ consisting of functions vanishing at infinity, and for every $u \in A_{p}(G),\|u\|_{\infty} \leq\|u\|_{A_{p}}$.

Denote $M A_{p}(G)=\left\{u \in C(G): u v \in A_{p}(G)\right.$ for all $\left.v \in A_{p}(G)\right\}$ with the norm $\|u\|_{M}=\sup \left\{\|u v\|_{A_{p}}: v \in A_{p}(G),\|v\|_{A_{p}} \leq 1\right\}$. It is called the space of multipliers of $A_{p}(G)$.

Let $V_{p}(G)=\left\{\psi: G \times G \rightarrow \mathbb{C}: \psi F \in L^{p}(G) \otimes_{\gamma} L^{q}(G)\right.$ for all $F \in L^{p}(G) \otimes_{\gamma}$ $\left.L^{q}(G)\right\}$. It is the space of pointwise multipliers of the projective tensor product $L^{p}(G) \otimes_{\gamma} L^{q}(G)$. The norm on $V_{p}(G)$ is the operator norm on $L^{p}(G) \otimes_{\gamma} L^{q}(G)$.

Let $\phi: G \rightarrow \mathbb{C}$ be a function. Define $M \phi: G \times G \rightarrow \mathbb{C}$ by

$$
M \phi(x, y)=\phi\left(x y^{-1}\right)
$$

for all $x, y \in G$. The space of Herz-Schur mulitipliers is defined to be

$$
B_{p}(G)=\left\{\phi: G \rightarrow \mathbb{C}: M \phi \in V_{p}(G)\right\} .
$$

The norm $\|\phi\|_{B_{p}}$ is given by $\|\phi\|_{B_{p}}=\|M \phi\|_{V_{p}}$. Elements of $B_{p}(G)$ are continuous, and $\|u\|_{\infty} \leq\|u\|_{B_{p}}$ for every $u \in B_{p}(G)$.

For each $1<p<\infty$, let $\mathcal{B}_{p}$ denote the category of $p$-spaces (see [21]). It is a subcategory of the category of Banach spaces. The following characterisation of the space $B_{p}(G)$ is due to Fendler [13, Theorem 4.4] (see also Pisier [29, Theorem 2.1] for a general treatment): a function $\phi$ on $G$ is in $B_{p}(G)$ if and only if there exist $B \in \mathcal{B}_{p}$ and (continuous) bounded maps $a: G \rightarrow B$ and $b: G \rightarrow B^{*}$ such that

$$
\phi\left(y x^{-1}\right)=\langle a(x), b(y)\rangle
$$

for all $x, y \in G$.

If $f \in C(G)$ and $x \in G$, then $\lambda(x) f$, the left translate of $f$ by $x$, is defined by $\lambda(x) f(y)=f\left(x^{-1} y\right) . \quad f \in C(G)$ is said to be a weakly almost periodic function (w.a.p. for short) if the set $\{\lambda(x) f ; x \in G\}$ is relatively compact with respect to the weak topology of $C(G)$. We denote by $W(G)$ the space of w.a.p. functions. It is known that $W(G)$ has a unique translation-invariant mean $m_{G}$. 
Finally, we point out that the inclusion $B_{p}(G) \subseteq W(G)(1<p<\infty)$ follows from the description of $B_{p}(G)$ and the Grothendieck criterion, which says that $f \in C(G)$ is w.a.p. if and only if whenever $\left\{x_{n}\right\}$ and $\left\{y_{m}\right\}$ are two sequences in $G$ and $\lim _{n} \lim _{m} f\left(x_{n} y_{m}\right)$ and $\lim _{m} \lim _{n} f\left(x_{n} y_{m}\right)$ exist, then they are equal.

\section{Discrete Groups}

Throughout this section, we will assume that $G$ is a discrete group.

First, let us give an alternative description of $B_{p}(G)$. Let $1<p<\infty$ and $\frac{1}{p}+\frac{1}{q}=1$, and let $E N D_{p}(G)$ be the Banach algebra of bounded linear operators on $l^{p}(G)$. Every element $k \in E N D_{p}(G)$ can be identified with a function $k: G \times G \rightarrow \mathbb{C}$ such that

$$
\|k\|_{E N D_{p}}=\sup \left\{\left|\sum_{x, y \in G} k(x, y) u(y) v(x)\right|:\|u\|_{p} \leq 1,\|v\|_{q} \leq 1\right\}
$$

is a finite number, where $u \in l^{p}(G)$ and $v \in l^{q}(G)$. The algebra of Herz-Schur multipliers $B_{p}(G)$ is the space of functions $\phi$ such that

$$
M \phi \cdot E N D_{p}(G) \subseteq E N D_{p}(G),
$$

where $M \phi \cdot k$ is the pointwise multiplication for $k \in E N D_{p}(G)$. The norm $\|\phi\|_{B_{p}}$ is given by

$$
\|\phi\|_{B_{p}}=\sup \left\{\|M \phi \cdot k\|_{E N D_{p}}:\|k\|_{E N D_{p}} \leq 1\right\} .
$$

Let $X_{p}$ be the completion of $l^{1}(G)$ with respect to the norm

$$
\|f\|_{X_{p}}=\sup \left\{\left|\sum_{x \in G} f(x) \phi(x)\right|: \phi \in B_{p}(G),\|\phi\|_{B_{p}} \leq 1\right\} .
$$

Then $X_{p}{ }^{*}=B_{p}(G)$, as was shown in [4], [13].

In [28], Picardello introduced the concept of weak Sidon sets, which was later made use of in [7]. In our situation, we need the following

Definition 3.1. A subset $S \subseteq G$ is said to be a $B_{p}$-Sidon set, if given any $f \in$ $l^{\infty}(G)$ there exists $u \in B_{p}(G)$ such that $\left.f\right|_{S}=\left.u\right|_{S}$.

If $g$ is a function defined on a subset $S$ of $G$, we can regard $g$ as a function on $G$ by setting its values to be zero outside of $S$. Thus, it is natural to identify $l^{p}(S)$ as a closed subspace of $l^{p}(G)$, for $1 \leq p \leq \infty$.

Proposition 3.2. Let $S$ be a subset of the discrete group $G$. Then the following conditions are equivalent:

(1) $S$ is a $B_{p}$-Sidon set;

(2) $l^{1}(S)$ is closed in $X_{p}$;

(3) $\|\cdot\|_{1}$ and $\|\cdot\|_{X_{p}}$ are equivalent on $l^{1}(S)$.

Proof. (1) $\Rightarrow(2)$. Suppose that $S$ is a $B_{p}$-Sidon set; then

$$
B_{p}(G) \stackrel{T}{\longrightarrow} l^{\infty}(S),\left.\quad u \longmapsto u\right|_{S},
$$

is continuous and surjective; hence $T$ is an open mapping. Therefore, there is a $\delta>0$ such that

$$
\operatorname{Ball}_{l^{\infty}(G)}(0, \delta) \subseteq T\left(\operatorname{Ball}_{B_{p}}(0,1)\right) .
$$


So for any $f \in l^{\infty}(G)$, there exists $u_{f} \in B_{p}(G)$ with $\left.f\right|_{S}=\left.u_{f}\right|_{S}$ and $\left\|\left.f\right|_{S}\right\|_{\infty} \geq$ $\frac{\delta}{2}\left\|u_{f}\right\|_{B_{p}}$.

Suppose that $\left\{g_{n}\right\}$ is a sequence in $l^{1}(S)$ that converges in the norm $\|\cdot\|_{X_{p}}$. For every $f \in L^{\infty}(S)$ with $\|f\|_{\infty}=1$, we have

$$
\begin{aligned}
\left|\left\langle g_{n}-g_{m}, f\right\rangle\right| & =\left|\left\langle g_{n}-g_{m},\left.u_{f}\right|_{S}\right\rangle\right| \\
& \leq\left\|g_{n}-g_{m}\right\|_{X_{p}}\left\|u_{f}\right\|_{B_{p}} \\
& \leq \frac{2}{\delta}\left\|g_{n}-g_{m}\right\|_{X_{p}} .
\end{aligned}
$$

So, $\left\{g_{n}\right\}$ is a Cauchy sequence in the norm $\|\cdot\|_{1}$, and hence $l^{1}(S)$ is closed in $X_{p}$.

$(2) \Rightarrow(1)$. Let $f \in l^{\infty}(S)=l^{1}(S)^{*}$. Since $l^{1}(S)$ is closed in $X_{p}$, we can get an extension $T \in X_{p}^{*}$ of $f$. Note that we can identify $T$ with a function $u \in B_{p}(G)$ by setting

$$
u(x)=T\left(\delta_{x}\right),
$$

where $\delta_{x}$ is the function on $G$ which is 1 at $x$ and 0 elsewhere. It is easy to see that $u(x)=f(x)$ holds for $x \in S$.

$(2) \Rightarrow(3)$. Note that $\|\cdot\|_{X_{p}} \leq\|\cdot\|_{1}$. So (3) is a consequence of the open mapping theorem.

(3) $\Rightarrow(2)$. Trivial.

We give another useful criterion of $B_{p}$-Sidon sets, similar to the Lemma 3.11 of [7].

Corollary 3.3. A subset $S$ of $G$ is a $B_{p}$-Sidon set if and only if there is a positive constant $c<1$ such that for every $f \in l^{\infty}(S)$ with $\|f\|_{\infty}=1$ there exists a $u \in$ $B_{p}(G)$ with $\left\|f-\left.u\right|_{S}\right\|_{\infty} \leq c$.

Proof. One direction is trivial.

Now suppose that $S$ is not a $B_{p}$-Sidon set. Then $\|\cdot\|_{1}$ and $\|\cdot\|_{X_{p}}$ are not equivalent on $l^{1}(S)$ by the above proposition. Let us choose $g_{1} \in l^{1}(S)$ with finite support $F_{1}$, and $\left\|g_{1}\right\|_{1}=1,\left\|g_{1}\right\|_{X_{p}}<1$. Note that $B_{p}(G)$ contains all functions with finite support; hence $S \backslash F_{1}$ is again not a $B_{p}$-Sidon set. Therefore, we can choose $g_{2} \in l^{1}\left(S \backslash F_{1}\right)$ with finite support $F_{2}$, and $\left\|g_{2}\right\|_{1}=1,\left\|g_{2}\right\|_{X_{p}}<\frac{1}{2}$. Continuing this procedure, we can get a sequence of functions $\left\{g_{n}\right\}$ in $l^{1}(S)$ with disjoint supports $F_{n}$, and $\left\|g_{n}\right\|_{1}=1,\left\|g_{n}\right\|_{X_{p}}<\frac{1}{n}$, for $n=1,2, \ldots$

Define

$$
f(x)= \begin{cases}\frac{g_{n}(x)}{\left|g_{n}(x)\right|}, & x \in F_{n} \text { for some } n, \\ 0, & x \notin \bigcup_{n=1}^{\infty} F_{n} .\end{cases}
$$

Then $f \in l^{\infty}(S)$ and $\|f\|_{\infty}=1$. Note that for every $u \in B_{p}(G)$,

$$
\begin{aligned}
\left\|f-\left.u\right|_{S}\right\|_{\infty} & \geq\left|\left\langle f-\left.u\right|_{S}, g_{n}\right\rangle\right| \\
& \geq\left|\left\langle f, g_{n}\right\rangle\right|-\left|\left\langle u, g_{n}\right\rangle\right| \\
& \geq 1-\|u\|_{B_{p}}\left\|g_{n}\right\|_{X_{p}} \\
& \geq 1-\frac{\|u\|_{B_{p}}}{n} .
\end{aligned}
$$

As $n$ can be arbitrarily large, $\left\|f-\left.u\right|_{S}\right\|_{\infty}=1$, and the condition in the statement is not satisfied. 
A subset $C$ of $G$ is called an $n$-square if $C=A B$ where $A, B \subseteq G$ and $|A|=$ $|B|=n$ and $|C|=n^{2}(|X|$ denotes the cardinality of the set $X)$. A subset $S$ of $G$ is said to contain large squares if for each positive integer $k, S$ contains a $k$-square.

Proposition 3.4. Suppose $S \subseteq G$ contains large squares. Then $\|\cdot\|_{1}$ and $\|\cdot\|_{X_{p}}$ are not equivalent on $l^{1}(S)$, i.e., $S$ is not a $B_{p}$-Sidon set.

Proof. For each integer $n>0$, choose an $n$-square $C=\left\{a_{1}, \ldots, a_{n}\right\}\left\{b_{1}, \ldots, b_{n}\right\}$.

It was shown by Bennett [1, Proposition 3.2] that there exist an $n \times n$ matrix $A=\left(a_{i j}\right)$ all of whose entries are \pm 1 , and a constant $D$, which is independent of $n$, such that the norm of the linear operator

$$
A: l^{p}\left(Z_{n}\right) \rightarrow l^{p}\left(Z_{n}\right),
$$

where $Z_{n}=\{1, \ldots, n\}$, satisfies

$$
\|A\|_{p, p} \leq \operatorname{Dmax}\left\{n^{\frac{1}{p}}, n^{\frac{1}{q}}\right\} .
$$

Let

$$
g=\sum_{i, j=1}^{n} a_{i j} \delta_{a_{i} b_{j}}
$$

then $g \in l^{1}(S)$ and $\|g\|_{1}=n^{2}$.

Now let us estimate $\|g\|_{X_{p}}$. By the definition,

$$
\|g\|_{X_{p}}=\sup \left\{\left|\sum_{x \in G} g(x) \phi(x)\right|: \phi \in B_{p}(G),\|\phi\|_{B_{p}} \leq 1\right\} .
$$

For $\phi \in B_{p}(G)$ with $\|\phi\|_{B_{p}} \leq 1$, we have

$$
\left|\sum_{x \in G} g(x) \phi(x)\right|=\left|\sum_{i, j=1}^{n} a_{i j} \phi\left(a_{i} b_{j}\right)\right| .
$$

Let $k \in E N D_{p}(G)$ be defined as

$$
k(x, y)= \begin{cases}a_{i j}, & \text { if }(x, y)=\left(a_{i}, b_{j}{ }^{-1}\right) \\ 0, & \text { otherwise }\end{cases}
$$

then

$$
\|k\|_{E N D_{p}}=\|A\|_{p, p} \leq \operatorname{Dmax}\left\{n^{\frac{1}{p}}, n^{\frac{1}{q}}\right\} .
$$

Let $k_{1}=M \phi \cdot k$ and $u=\sum_{j=1}^{n} \delta_{b_{j}^{-1}}$; then for every $v \in l^{q}(G)$,

$$
\left|\sum_{x, y \in G} k_{1}(x, y) u(y) v(x)\right| \leq\left\|k_{1}\right\|_{E N D_{p}}\|u\|_{p}\|v\|_{q},
$$

i.e.,

$$
\left|\sum_{i, j=1}^{n} a_{i j} \phi\left(a_{i} b_{j}\right) v\left(a_{i}\right)\right| \leq n^{\frac{1}{p}}\left\|k_{1}\right\|_{E N D_{p}}\|v\|_{q} .
$$

Therefore, we get

$$
\left(\sum_{i=1}^{n}\left|\sum_{j=1}^{n} a_{i j} \phi\left(a_{i} b_{j}\right)\right|^{p}\right)^{\frac{1}{p}} \leq n^{\frac{1}{p}}\left\|k_{1}\right\|_{E N D_{p}} .
$$


Hence

$$
\begin{aligned}
\left|\sum_{i, j=1}^{n} a_{i j} \phi\left(a_{i} b_{j}\right)\right| & \leq \sum_{i=1}^{n}\left|\sum_{j=1}^{n} a_{i j} \phi\left(a_{i} b_{j}\right)\right| \\
& \leq n^{\frac{1}{q}}\left(\sum_{i=1}^{n}\left|\sum_{j=1}^{n} a_{i j} \phi\left(a_{i} b_{j}\right)\right|^{p}\right)^{\frac{1}{p}} \\
& \leq n\left\|k_{1}\right\|_{E N D_{p}} \\
& \leq n\|k\|_{E N D_{p}}\|\phi\|_{B_{p}} \\
& \leq D \max \left\{n^{1+\frac{1}{p}}, n^{\frac{1}{q}}\right\}
\end{aligned}
$$

So,

$$
\|g\|_{X_{p}} \leq \operatorname{Dmax}\left\{n^{1+\frac{1}{p}}, n^{1+\frac{1}{q}}\right\}
$$

Since $n$ can be arbitrarily large, we conclude that $\|\cdot\|_{1}$ and $\|\cdot\|_{X_{p}}$ are not equivalent.

A subset $T \subseteq G$ is said to be a $t$-set if $(T \cap T x) \cup(T \cap x T)$ is finite for every $x \in G \backslash\{e\}$. It is known that if $T$ is a $t$-set, then any $f \in l^{\infty}(G)$ with $\operatorname{supp} f \subseteq T$ is in $W(G)$; see [6], [7].

A consequence of Proposition 3.4 related to the concept of $t$-set is the following:

Corollary 3.5. If $S$ is a countable $B_{p}$-Sidon set, then $S$ is a finite union of $t$-sets.

Proof. Since $S$ does not contain large squares, by Theorem 4.1 of [8] $S$ is a finite union of $t$-sets.

The following result of Chou [7] will play a very important role in our proof of the main result of this section.

Theorem (Chou). If $G$ is an infinite group, then there is a $t$-set $T$ of $G$ such that $T=\bigcup_{n=1}^{\infty} S_{n}$ is a disjoint union and each $S_{n}$ contains large squares.

By applying Proposition 3.4 and a device in Chou [7], we are able to show the following.

Theorem 3.6. Let $G$ be an infinite discrete group. Then $W(G) / B_{p}(G)^{-}$contains a linear isometric copy of $l^{\infty}(\mathbb{N})$. In particular, $B_{p}(G)^{-}$is a proper subset of $W(G)$.

Proof. Let $T$ be the $t$-set as in Chou's construction. So $T=\bigcup_{n=1}^{\infty} S_{n}$ is a disjoint union of $S_{n}$ 's with each $S_{n}$ containing large squares. Thus each $S_{n}$ is not a $B_{p^{-}}$ Sidon set, and hence by Corollary 3.3, there exists a function $f_{n} \in l^{\infty}(G)$ with the following properties: $\left\|f_{n}\right\|_{\infty}=1, \operatorname{supp}_{n} \subseteq S_{n}$ and $\left\|\left.\left(f_{n}-u\right)\right|_{S_{n}}\right\|_{l^{\infty}\left(S_{n}\right)} \geq 1$ for every $u \in B_{p}(G)$.

Since $S_{n}$ is a $t$-set, $f_{n} \in W(G), n=1,2, \ldots$.

Define

$$
\begin{gathered}
\xi: l^{\infty}(\mathbb{N}) \rightarrow W(G) / B_{p}(G)^{-}, \\
\left(c_{n}\right) \mapsto \sum_{i=1}^{\infty} c_{n} f_{n}+B_{p}(G)^{-} .
\end{gathered}
$$

It is not hard to see that $\xi$ is an isometry. 


\section{Nilpotent Groups and [IN]-Groups}

First, let us recall that a locally compact group $G$ is called an [IN]-group if it has a compact neighborhood of the identity which is invariant under all inner automorphisms of $G$.

Let $H$ be a closed normal subgroup of $G$ and

$$
\pi: G \rightarrow G / H
$$

be the canonical homomorphism. For $1<p<\infty$ and $f \in B_{p}(G / H)$, there exist $B \in \mathcal{B}_{p}$ and continuous bounded maps $a_{0}: G / H \rightarrow B, b_{0}: G / H \rightarrow B^{*}$ such that

$$
f\left(\pi(y) \pi(x)^{-1}\right)=\left\langle a_{0}(\pi(x)), b_{0}(\pi(y))\right\rangle
$$

for all $x, y \in G$. Let $a=a_{0} \circ \pi, b=b_{0} \circ \pi$; then the continuous function $f \circ \pi$ on $G$ satisfies

$$
(f \circ \pi)\left(y x^{-1}\right)=\langle a(x), b(y)\rangle
$$

for all $x, y \in G$. So, $f \circ \pi \in B_{p}(G)$ and the map

$$
\begin{gathered}
\Phi: B_{p}(G / H) \rightarrow B_{p}(G), \\
f \mapsto f \circ \pi,
\end{gathered}
$$

is an isometry from $B_{p}(G / H)$ onto the subspace of $B_{p}(G)$ consisting of functions that are constant on the left cosets of $H$.

Fix $x \in G$. For any function $f$ on $G$, define a function

$$
f_{x}: H \rightarrow \mathbb{C}
$$

by $f_{x}(t)=f(x t), t \in H$. If $f \in B_{p}(G)$ and

$$
f\left(y x^{-1}\right)=\langle a(x), b(y)\rangle
$$

for some space $B \in \mathcal{B}_{p}$ and bounded maps $a: G \rightarrow B, b: G \rightarrow B^{*}$, then

$$
f_{x}\left(t s^{-1}\right)=f\left(x t s^{-1}\right)=\langle a(s), b(x t)\rangle
$$

for $s, t \in H$; so $f_{x} \in B_{p}(H)$.

Let $m_{H}$ be the unique invariant mean of $W(H)$. For $f \in B_{p}(G)$ and $x \in G$; since $f_{x} \in B_{p}(H) \subseteq W(H)$, we can define

$$
\phi(x)=m_{H}\left(f_{x}\right)
$$

for $x \in G$.

Proposition 4.1. Let $\phi$ be defined as above. Then $\phi \in B_{p}(G)$ and $\phi$ is constant on the left cosets of $H$.

Proof. Since $m_{H}$ is $H$-invariant, $\phi$ is constant on left cosets of $H$. The function $\phi$ is continuous, since $x \mapsto f_{x}$ is continuous.

By a result of Davis [10], there exists a net of open and relatively compact subsets $\left\{U_{\alpha}\right\}$ of $H$ such that

$$
m_{H}(k)=\lim _{\alpha} \lambda\left(U_{\alpha}\right)^{-1} \int_{U_{\alpha}} k(t) d \lambda(t),
$$

where $k \in W(H)$ and $\lambda$ is a fixed left Haar measure of $H$. 
Let $B \in \mathcal{B}_{p}$. If $p: H \rightarrow B$ or $B^{*}$ is a continuous bounded map, $U \subseteq H$ is a relatively compact open set, the vector-valued integral

$$
\int_{U} p(t) d \lambda(t)
$$

exists, and $\left\|\int_{U} p(t) d \lambda(t)\right\| \leq \int_{U}\|p(t)\| d \lambda(t)$.

For fixed $x, y \in G$,

$$
\begin{aligned}
\phi\left(y x^{-1}\right) & =m_{H}\left(f_{y x^{-1}}\right) \\
& =\lim _{\alpha} \frac{1}{\lambda\left(U_{\alpha}\right)} \int_{U_{\alpha}} f\left(y x^{-1} t\right) d \lambda(t) \\
& =\lim _{\alpha} \frac{1}{\lambda\left(U_{\alpha}\right)} \int_{U_{\alpha}}\left\langle a\left(t^{-1} x\right), b(y)\right\rangle d \lambda(t) \\
& =\lim _{\alpha}\left\langle c_{\alpha}(x), b(y)\right\rangle
\end{aligned}
$$

where

$$
c_{\alpha}(x)=\frac{1}{\lambda\left(U_{\alpha}\right)} \int_{U_{\alpha}} a\left(t^{-1} x\right) d \lambda(t)
$$

Note that

$$
\begin{aligned}
\left\|c_{\alpha}(x)\right\| & \leq \frac{1}{\lambda\left(U_{\alpha}\right)} \int_{U_{\alpha}}\left\|a\left(t^{-1} x\right)\right\| d \lambda(t) \\
& \leq \sup _{x \in G}\|a(x)\|,
\end{aligned}
$$

and that any space in $\mathcal{B}_{p}$ is reflexive [21, Proposition 7], the net $\left\{c_{\alpha}(x)\right\}$ has a weak limit, say $c(x)$, in $B$. Clearly, $\|c(x)\| \leq \sup _{x \in G}\|a(x)\|$ and

$$
\phi\left(y x^{-1}\right)=\langle c(x), b(y)\rangle
$$

for all $x, y \in G$. So $\phi \in B_{p}(G)$.

Let $A=\left(a_{i j}\right)_{n \times n}, B=\left(b_{i j}\right)_{n \times n}$ be two matrices. The Schur product of $A$ and $B$ is the matrix

$$
A * B=\left(a_{i j} b_{i j}\right)_{n \times n} .
$$

Let $\|A\|_{(p)}=\sup \left\{\|A * B\|_{p, p}:\|B\|_{p, p} \leq 1\right\}$. Recall that $\|\cdot\|_{p, p}$ is the norm of a linear operator on $l^{p}\left(Z_{n}\right)$.

We will use the following characterisation of $B_{p}(G)$ due to Fendler [13]:

Lemma. A function $\phi$ on $G$ is in $B_{p}(G)$ if and only if $\phi$ is continuous and there is a constant $C$ such that for any finite set $\left\{x_{1}, \ldots, x_{n}\right\} \subseteq G,\left\|\left(\phi\left(x_{i} x_{j}^{-1}\right)\right)_{n \times n}\right\|_{(p)} \leq C$.

In order to prove the main result of this section, we need the next lemma.

Lemma 4.2. Let $H$ be an open subgroup of $G$. Extend $f \in C(H)$ to $f^{\circ} \in C(G)$ by setting $f^{\circ}(x)=0$ if $x \in G \backslash H$. If $f \in B_{p}(H)$, then $f^{\circ} \in B_{p}(G)$.

Proof. $f^{\circ}$ is clearly a continuous function on $G$. Since $f \in B_{p}(H)$, there exists a constant $C$ such that for any finite set $\left\{t_{1}, \ldots, t_{k}\right\} \subseteq H,\left\|\left(f\left(t_{j} t_{j}^{-1}\right)\right)_{k \times k}\right\|_{(p)} \leq C$.

Consider now a finite set $\left\{x_{1}, \ldots, x_{n}\right\} \subseteq G$ of cardinality $n$. Since the norm of a matrix remains the same after interchanging any two rows or any two columns, we may assume that $x_{1}, \ldots, x_{l}$ belong to a right coset of $H$ and $x_{l+1}, \ldots, x_{n}$ belong to another right coset of $H$ (the proof for the case of more cosets is similar). 
Let $A=\left(a_{i j}\right)$ be an $n \times n$ matrix with $\|A\|_{p, p} \leq 1$. Then

where

$$
\left(f^{\circ}\left(x_{i} x_{j}^{-1}\right)\right)_{n \times n} * A=\left(\begin{array}{cc}
T_{1} * A_{1} & 0 \\
0 & T_{2} * A_{2}
\end{array}\right)
$$

$$
A_{1}=\left(\begin{array}{ccc}
a_{11} & \cdots & a_{1 l} \\
\cdot & \cdots & \cdot \\
a_{l 1} & \cdots & a_{l l}
\end{array}\right), \quad A_{2}=\left(\begin{array}{ccc}
a_{l+1, l+1} & \cdots & a_{l+1, n} \\
\cdot & \cdots & \cdot \\
a_{n, l+1} & \cdots & a_{n n}
\end{array}\right)
$$

and

$$
T_{1}=\left(\begin{array}{ccc}
f\left(x_{1} x_{1}^{-1}\right) & \cdots & f\left(x_{1} x_{l}^{-1}\right) \\
\cdot & \cdots & \cdot \\
f\left(x_{l} x_{1}^{-1}\right) & \cdots & f\left(x_{l} x_{l}^{-1}\right)
\end{array}\right), \quad T_{2}=\left(\begin{array}{ccc}
f\left(x_{l+1} x_{l+1}^{-1}\right) & \cdots & f\left(x_{l+1} x_{n}^{-1}\right) \\
\cdot & \cdots & \cdot \\
f\left(x_{n} x_{l+1}^{-1}\right) & \cdots & f\left(x_{n} x_{n}^{-1}\right)
\end{array}\right) .
$$

Note that

$$
\left\|A_{1}\right\|_{p, p} \leq 1, \quad\left\|A_{2}\right\|_{p, p} \leq 1
$$

so

$$
\left\|T_{1} * A_{1}\right\|_{p, p} \leq C, \quad\left\|T_{2} * A_{2}\right\|_{p, p} \leq C .
$$

Since $\left(f^{\circ}\left(x_{i} x_{j}^{-1}\right)\right)_{n \times n} * A$ is in diagonal form, we have the following equality:

$$
\left\|\left(f^{\circ}\left(x_{i} x_{j}^{-1}\right)\right)_{n \times n} * A\right\|_{p, p}=\max \left\{\left\|T_{1} * A_{1}\right\|_{p, p},\left\|T_{2} * A_{2}\right\|_{p, p}\right\} .
$$

Since $A$ can be arbitrary, we conclude that

$$
\left\|\left(f^{\circ}\left(x_{i} x_{j}^{-1}\right)\right)_{n \times n}\right\|_{(p)} \leq C .
$$

Hence, by the above lemma, $f^{\circ} \in B_{p}(G)$.

Applying Proposition 4.1, Lemma 4.2 and Theorem 3.6, a proof similar to that of Theorem 4.5 of Chou [7] gives us

Theorem 4.3. Let $G$ be a noncompact nilpotent group or a noncompact [IN]-group. Then $W(G) / B_{p}(G)^{-}$contains a linear copy of $l^{\infty}(\mathbb{N})$. In particular, $B_{p}(G)^{-}$is a proper subset of $W(G)$.

\section{Free Groups}

It is well known that for an amenable locally compact group $G$, and $1<p<\infty$, $M A_{p}(G)=B_{p}(G)$, and in particular, $M A(G)=M_{0} A(G)=B(G)$. Losert [26] showed that $M A(G)=B(G)$ implies the amenability of $G$ (the discrete case was due to Nebbia [27]), and for a discrete group $G$, Bożejko [3] showed that $M_{0} A(G)=$ $B(G)$ implies the amenability of $G$. He also obtained in [2], [4] the following result: for a noncommutative free group $F, B_{p}(F) \subsetneq M A_{p}(F)$. The proof in [4] gives a function $\phi$ with $\phi \in M A(F)$ but $\phi \notin W(F)$, hence $\phi \notin B_{p}(F)$. Thus it is natural to ask whether $B_{p}(F)=M A_{p}(F) \cap W(F)$. In this section, we show that this is not the case.

Recall that a subset $E$ of a discrete group $G$ is a Leinert set if there is $C>0$ such that for every $f \in l^{2}(E)$

$$
\|f\|_{V N}=\sup \left\{\|f * g\|_{2}:\|g\|_{2}=1\right\} \leq C\|f\|_{2},
$$


or, equivalently,

$$
\chi_{E} A(G)=l^{2}(E) .
$$

It was shown by Bożejko that $E$ is a Leinert set if and only if $l^{\infty}(E) \subseteq M A(G)$, and if $E$ is a Leinert set then $l^{\infty}(E) \subseteq M A_{p}(G)$, for $1<p<\infty$; see [2]. Now we are ready for the main result of this section.

Theorem 5.1. Let $F$ be the free group on $k$ generators with $k>1$. Then $B_{p}(F)$ is a proper subset of $M A_{p}(F) \cap W(F)$.

Proof. First, let us consider the case that $k=\infty$. Let $E=\left\{x_{1}, x_{2}, \cdots\right\}$ be the set of free generators of $F$. By the Haagerup convolution theorem [18] (see also [14]), we conclude that $E^{2}=\left\{x_{i} x_{j}: i, j=1,2, \cdots\right\}$ is a Leinert set.

For an integer $k>0$, define

$$
T_{k}=\left\{x_{i} x_{j} \in E^{2}: 2^{k-1} \leq i, j<2^{k}\right\}
$$

and set

$$
T=\bigcup_{k=1}^{\infty} T_{k}
$$

Since $T$ contains large squares, by Proposition 3.4 it is not a $B_{p}$-Sidon set. Therefore, by Corollary 3.3, we can find a function $\phi \in l^{\infty}(F)$ such that $\operatorname{supp} \phi \subseteq T$, $\|\phi\|_{\infty}=1$ and $\left\|\phi-\left.u\right|_{T}\right\|_{l^{\infty}(T)} \geq 1$ for every $u \in B_{p}(F)$. In particular, $\phi \notin B_{p}(F)$. We claim that $\phi \in M A_{p}(F) \cap W(F)$.

$\phi \in M A_{p}(F)$, since supp $\phi \subseteq T$ and $T$ is a Leinert set, being a subset of the Leinert set $E^{2}$.

To show that $\phi \in W(F)$, it suffices to show that $T$ is a $t$-set. Indeed, let $x \in F \backslash\{e\}$ and $x=x_{i_{1}}^{u_{1}} \cdots x_{i_{n}}^{u_{n}}$ be the reduced form, where $u_{i}= \pm 1, i=1, \cdots, n$. If $y \in T \cap x T$, then

$$
y=x_{i} x_{j} \in T_{k}
$$

for some positive integer $k$, and

$$
y=x_{i_{1}}^{u_{1}} \cdots x_{i_{n}}^{u_{n}} x_{u} x_{v}
$$

for some $x_{u} x_{v} \in T$.

Comparing the two forms of $y$, and noticing that $x \neq e$, we get $i=i_{1}$. Moreover, $x$ can take the forms $x_{i_{1}}^{u_{1}} x_{i_{2}}^{u_{2}} x_{i_{3}}^{u_{3}} x_{i_{4}}^{u_{4}}$ and $x_{i_{1}}^{u_{1}} x_{i_{2}}^{u_{2}}$. In the first case, we have at most one choice of $y$, namely $y=x_{i_{1}} x_{i_{2}}$, provided $u_{1}=u_{2}=1$ and $x_{i_{3}}^{u_{3}} x_{i_{4}}^{u_{4}} x_{u} x_{v}=e$. In the second case, let $k$ be $\left[\log _{2} i_{1}\right]+1$; then we have at most $2^{k-1}$ choices of $y$, namely $y=x_{i_{1}} x_{j}$ with $j=2^{k-1}, 2^{k-1}+1, \ldots, 2^{k}-1$, provided $u_{2}=-1, i_{2}=u$. So

$$
|T \cap x T| \leq i_{1}+1<\infty \text {. }
$$

Similarly , $|T \cap T x|<\infty$.

Now let $F$ be the free group on $k$ generators with $k>1$. We can find a subgroup $H$ of $F$ with $H \cong F_{\infty}$. Therefore, there exists a function $\phi \in W(H) \cap M A_{p}(H)$, but $\phi \notin B_{p}(H)$, as in the proof above.

Let us extend $\phi$ to a function $\phi^{0}$ on $F$ by setting $\phi^{0}(x)=0$ for $x \notin H$. Using the definition of Herz-Schur multipliers, we can check that $\phi^{0} \notin B_{p}(F)$. Also, by Lemma 4.1 of [7], $\phi^{0} \in W(F)$. Notice that our $\phi$ has support in a Leinert set $E$ of $H$. To show $\phi \in M A_{p}(F)$, it suffices to show that $E$ is a Leinert set of $F$. By a theorem of Herz [22], $\left.u\right|_{H} \in A(H)$ whenever $u \in A(F)$. Let $u \in A(F)$ since $E$ is a 
Leinert set of $H,\left.\left(\chi_{E} u\right)\right|_{H} \in l^{2}(H)$. Hence $\chi_{E} u \in l^{2}(F)$, which shows that $E$ is a Leinert set of $F$.

So, $\phi^{0} \in M A_{p}(F) \cap W(F) \backslash B_{p}(F)$, and the proof is complete.

\section{ACKNowledgement}

This paper is a part of the author's Ph.D. dissertation at SUNY Buffalo. He expresses his deepest gratitude to his advisor, Professor C. Chou, for guidance and encouragement. The author is indebted to Professor J. Kraus for valuable conversations. The author is very grateful to Professor E. E. Granirer the comments and encouragement. Thanks also go to Professor M. Bożejko for providing [4] and to Dr. G. Fendler for providing [13] to the author. Finally, the author wants to thank the referee for suggesting the present version of Theorem 4.3. The auther's original version dealt only with the case of $p=2$.

\section{REFERENCES}

[1] G. Bennett, Schur multipliers, Duke Math. J. 44 (1977), 603-639. MR 58:12490

[2] M. Bożejko, Remark on Herz-Schur multipliers on free groups, Math. Ann. 258 (1981), 11-15. MR 83a: 43001

[3] - Positive definite bounded matrices and a characterization of amenable groups, Proc. Amer. Math. Soc. 95 (1985), 357-359. MR 87h:43006

[4] _ Positive and negative definite kernels on discrete groups, Lectures at Heidelberg Univ., 1987.

[5] M. Bożejko and G. Fendler, Herz-Schur multipliers and completely bounded multipliers of Fourier algebra of a locally compact group, Boll. Un. Mat. Ital. (6)3-A (1984), 297-302. MR 86b:43009

[6] R. B. Burckel, Weakly almost periodic functions on semigroups, Gordon and Breach, New York, 1970. MR 41:8562

[7] C. Chou, Weakly almost periodic functions and Fourier-Stieltjes algebras of locally compact groups, Trans. Amer. Math. Soc. 274 (1982), 141-157. MR 84a:43008

[8] - Weakly almost periodic functions and thin sets in discrete groups, Trans. Amer. Math. Soc. 321 (1990), 333-346. MR 90m:43023

[9] M. Cowling and U. Haagerup, Completely bounded multipliers of the Fourier algebra of a simple Lie group of real rank one, Invent. Math. 96 (1989), 507-549. MR 90h:22008

[10] H. W. Davis, Generalized almost periodicity in groups, Trans. Amer. Math. Soc. 191 (1974), 329-352. MR 50:898

[11] J. De Cannière and U. Haagerup, Multipliers of the Fourier algebra of some simple Lie groups and their discrete subgroups, Amer. J. Math. 107 (1984), 455-500. MR 86m:43002

[12] P. Eymard, L'algèbre de Fourier d'un groupe localement compact, Bull. Soc. Math. France 92 (1964), 181-236. MR 37:4208

[13] G. Fendler, Herz Schur multipliers and coefficients of bounded representations, Thesis at Heidelberg Univ., 1987.

[14] A. Figà-Talamanca and M. A. Picardello, Harmonic analysis on free groups, Lecture Notes in Pure and Appl. Math., vol. 87, Marcel Dekker, New York, 1983. MR 85j:43001

[15] K. Furuta, Algebras $A_{p}$ and $B_{p}$ and amenability of locally compact groups, Hokkaido Math. J. 20 (1991), 579-591. MR 92m:43004

[16] E. E. Granirer, Some results on $A_{p}(G)$ submodules of $P M_{p}(G)$, Colloq. Math. 51 (1987), 155-163. MR 88f: 43008

$[17] \_$, On some spaces of linear functionals on the algebras $A_{p}(G)$ for locally compact groups, Colloq. Math. 52 (1987), 119-132. MR 88k:43006

[18] U. Haagerup, An example of a nonnuclear $C^{*}$-algebra which has the metric approximation property, Invent. Math. 50 (1979), 279-293. MR 80j:46094

[19] - Group $C^{*}$-algebra without the completely bounded approximation property, Preprint.

[20] U. Haagerup and J. Kraus, Approximation properties for group $C^{*}$-algebras and group von Neumann algebras, Trans. Amer. Math. Soc. 344 (1994), 667-699. MR 94k:22008 
[21] C. Herz, The theory of p-spaces with an application to convolution operators, Trans. Amer. Math. Soc. 154 (1971), 69-82. MR 42:7833

[22] — Harmonic synthesis for subgroups, Ann. Inst. Fourier (Grenoble) 23 (1973), 91-123. MR 50:7956

[23] _ Une généralization de la notion de transformée de Fourier-Stieltjes, Ann. Inst. Fourier (Grenoble) 24 (1974), 145-157. MR 54:13466

[24] A. T. Lau and A. Ülger, Some geometric properties on the Fourier algebras and Fourier Stieltjes algebras of locally compact groups, Arens regularity and related problems, Trans. Amer. Math. Soc. 337 (1993), 321-359. MR 93g:22007

[25] M. Leinert, Faltungsoperatoren auf gewissen diskreten Gruppen, Studia Mat. 62 (1973), 149158. MR 50:7954

[26] V. Losert, Properties of the Fourier algebra that are equivalent to amenability, Proc. Amer. Math. Soc. 92 (1984), 347-354. MR 86b:43010

[27] C. Nebbia, Multipliers and asymptotic behaviour of the Fourier algebra of nonamenable groups, Proc. Amer. Math. Soc. 84 (1982), 549-554. MR 83h:43002

[28] M. A. Picardello, Lacunary sets in discrete noncommutative groups, Bull. Un. Mat. Ital. 8 (1973), 494-508. MR 49:9543

[29] G. Pisier, Completely bounded maps between sets of Banach space operators, Indiana Univ. Math. J. 39 (1990), 249-277. MR 91k:47078

[30] _ Multipliers and lacunary sets in non-amenable groups, Amer. J. Math. 117 (1995), 337-376. MR 96e:46078

[31] D. Ramirez, Weakly almost periodic functions and Fourier-Stieltjes transforms, Proc. Amer. Math. Soc. 19 (1968), 1087-1088. MR 38:488

[32] W. Rudin, Weakly almost periodic functions and Fourier-Stieltjes transforms, Duke Math J. 26 (1959), 215-220. MR 21:1492

Department of Mathematics, State University of New York at Buffalo, Buffalo, NEW YORK 14214-3093

Current address: Department of Mathematical Sciences, University of Alberts, Edmonton, AB, T6G 2G1, Canada

E-mail address: gxu@vega.math.ualberta.ca 